\title{
Recycling und Wiederverwendung von Fasern aus Abfällen
}

Zusammenfassung: Die Menge an zu entsorgenden Fasern ist im Vergleich zum Gesamtaufkommen an Abfall gering. Da jedoch die Herstellung von Fasern aufwändige und teuere Prozesse erfordert, so kann deren Recycling sowohl aus ökologischen als auch aus ökonomischen Überlegungen Sinn machen. Da durch verschärfte gesetzliche Rahmenbedingungen innerhalb der EU eine Abfallentsorgung zunehmend verteuert wird, können sich die Kosten für eine weitere Aufbereitung der Altfasern rechnen. Dies kann dann gelingen, wenn das aufbereitete Produkt zu einem attraktiven Marktpreis abzusetzen ist. Für eine erfolgreiche Umsetzung von Faserrecycling muss eine geeignete $\mathrm{Ab}$ fallfraktion gefunden, der Aufbereitungsprozess adaptiert und gewinnbringende Anwendungen entwickelt werden.

\section{Recycling fibres from waste}

Summary: Fibres to be disposed of account for a relatively small proportion of the total amount of waste production. Since the production of fibres involves expensive processes, however, their recycling can make sense, both ecologically and economically. With waste disposal becoming more and more expensive as a result of a tightening legal situation within the EU, processing waste fibres may pay off. This will in fact be profitable if and when the processed product can be sold at an attractive market price. For fibre recycling to become worthwhile, it is first necessary to find a suitable waste fraction, adapt the processing method and develop profitable applications.

\section{Rahmenbedingungen für Abfälle}

Der Umgang mit Abfällen hat sich in den letzten Jahrzehnten dramatisch verändert. Bis in die 1960er Jahre stellte die Müllbehandlung primär eine Gesundheitsmaßnahme dar, die sicher stellte, dass Abfälle aus der Stadt gebracht wurden, um die Seuchengefahr zu bannen. Dabei wurden Abfälle weitgehend in Schotter- und Kies- gruben abgelagert. Erst in den 1970er und 1980er Jahren begann in Österreich eine sachgemäße Entsorgung, die primär auf einer Sammlung und einer geordneten Deponierung beruhten. Dabei kam erstmalig der Begriff Umweltschutz für die Behandlung von Abfall zum Tragen. Eine umfassende und flächendeckende getrennte Sammlung setzte sich in Österreich erst in den 1990er Jahren aufgrund der Verpackungsverordnung durch. Dies resultierte in einer Etablierung von Kreislaufsystemen, die bei Glas und Papier zu beachtlichen Recyclingquoten führen. In den letzten Jahren trat in Österreich, aber auch in Deutschland ein Deponierungsverbot für unbehandelte Abfälle in Kraft, wodurch die Abfallströme in Richtung thermische und mechanisch-biologische Verwertungsanlagen gelenkt wurden. Damit war eine deutliche Kostensteigerung für die Abfallentsorgung verbunden.

Für faserhältige Abfälle gelten natürlich die gleichen Verordnungen und Richtlinien. Derzeit werden diese Produkte noch großteils deponiert oder in Ländern wie Österreich und Deutschland aufgrund des Deponierungsverbotes thermisch entsorgt. Da die Herstellung von Fasern jedoch sehr aufwändig und teuer ist, könnten durch eine Wiederverwendung von Altfasern sowohl Kosten gespart werden, als auch der Verbrauch an Ressourcen und Energie markant verringern werden. Allerdings sind Recyclingprozesse für Altfasern noch nicht sehr verbreitet und müssen vielfach erst entwickelt werden.

\section{Fasermarkt}

Prinzipiell unterscheidet man zwischen Natur- und Kunstfasern. Die Naturfasern ihrerseits werden in zwei Gruppen geteilt, je nachdem ob diese pflanzlichen oder tierischen Ursprungs sind. Kunstfasern entstehen durch Verspinnen von Polymeren, die entweder aus synthetischen oder natürlichen Rohstoffen stammen. Synthetische Polymere werden aus Erdöl gewonnen und sind daher prinzipiell nicht als nachhaltiges Produkt anzusehen. Zellulose, die aus Holz oder anderen Pflanzen gewonnen wird, ist das einzig relevante natürliche Polymer, das zur Herstellung von Fasern verwendet wird.

Die jährliche Produktion von Fasern lag im Jahre 2007 bei 73,7 Million t (CIRFS, 2008). Fasern aus synthetischen Polymeren machen mit 58,1\% (42,8 Millionen $\mathrm{t}$ ) den größten Anteil aus. Die zweitwichtigste Gruppe stellen die Naturfasern mit $37,1 \%$ (27,3 Millionen t) dar, unter denen Bauwolle die mit Abstand bedeutendste Faser ist (26,1 Millionen t). Zellulosische Fasern sind mit 3,6 Millionen t $(4,8 \%)$ mengenmäßig von untergeordneter Bedeutung (CIRFS 2008). In dieser Statistik fehlen jedoch die Glasfasern, deren Jahresproduktion etwa bei 3 Millionen $t$ liegt.

Die Verwendung von Fasern ist vielfältig und die Einsatzgebiete teilen sich auf die Hauptgruppen Bekleidung, Heimtextilien und technische Anwendungen auf Eine genaue quantitative Zuordnung der einzelnen Marksegmente ist nicht möglich, da insbesondere aus dem asiatischen Raum kaum brauchbare Daten verfügbar sind. Es ist jedoch klar, dass, aufgrund des breiten Einsatzspektrums, Fasern in den unterschiedlichsten Abfallfraktionen zu finden sind und die Menge an Altfasern, mit einer zeitlichen Verzögerung, in etwa der Produktionsmenge entspricht.

\section{Einsparpotentiale}

Der Verbrauch von Ressourcen und Energie bei der Herstellung von Fasern ist, abhängig von der chemischen Natur, sehr unterschiedlich. Auch wenn Baumwolle zu den nachwachsenden Rohstoffen zählt, so kann deren Anbau und Produktion keinesfalls als nachhaltig angesehen werden. Der Anbau von Baumwolle beanspruch etwa $340000 \mathrm{~km}^{2}$. Das entspricht etwa der Größe von Deutschland oder rund 2,4\% der weltweit verfügbaren Ackerfläche. $\mathrm{Ob}$ wohl die Anbaufläche seit den letzten 20 Jahren stagnierte, stieg die Produktion jährlich um durchschnittlich 2,2\%. Diese Steigerung basiert primär auf einer starken Zunahme des Einsatzes von Dünger, Bewässerung und Schädlingsbekämpfungsmittel. Schätzungen zufolge werden zwischen 5,8 und $18 \%$ der weltweiten Pestizidproduktion ausschließlich für den 


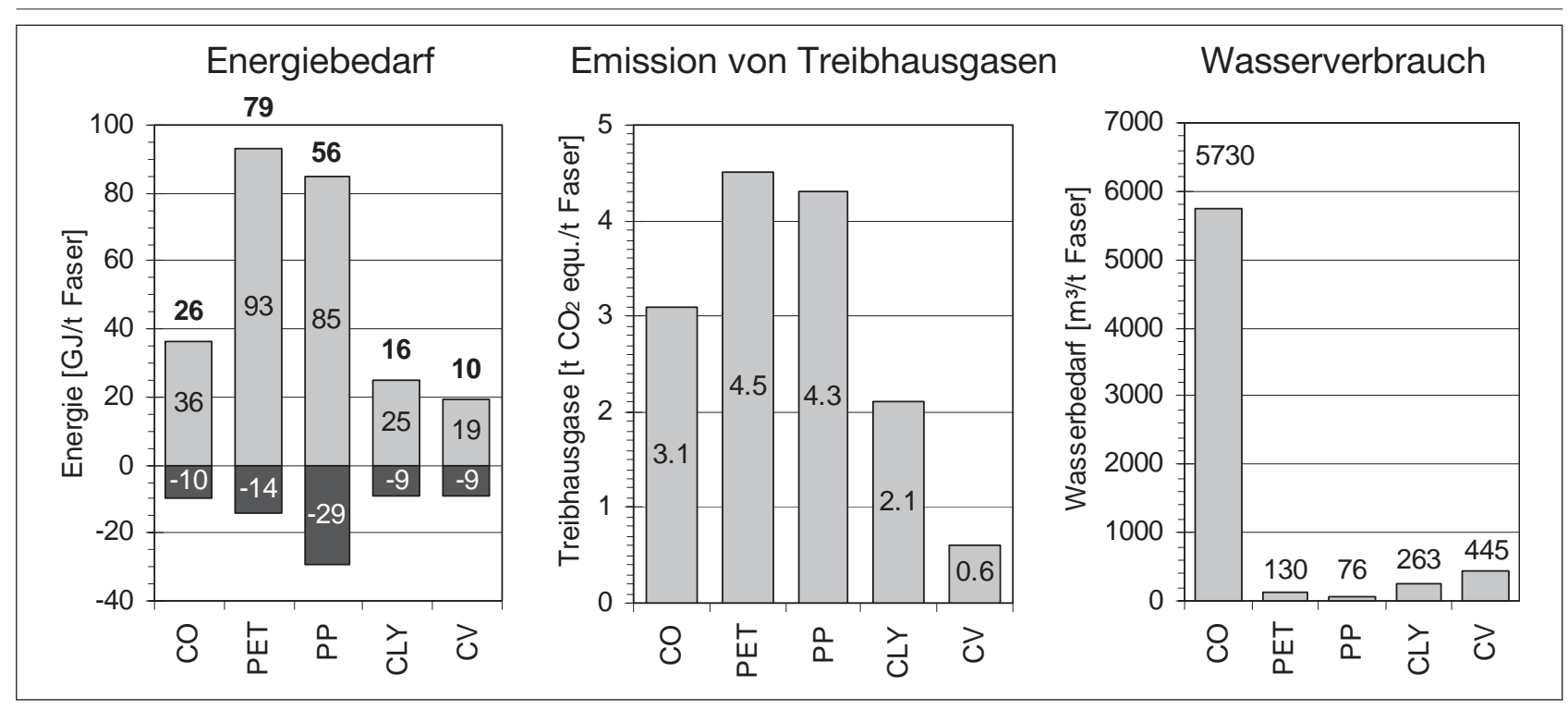

Abb. 1: Energiebedarf, Emissionen von Treibhausgasen und Wasserbedarf bei der Herstellung verschiedener Fasern. Die negativen Werte ergeben sich aus der Annahme, dass die Fasern thermisch entsorgt werden, wobei $60 \%$ der Energie Rück gewonnen wird (Patel und Shen 2008); Kurzzeichen der Fasern gemäß (Norm 1999; BISFA 2000).

Baumwollanbau eingesetzt (Paulitsch et al. 2004).

Tabelle 1 vergleicht den Energiebedarf, die Emissionen von Treibhausgasen und den Wasserbedarf bei der Herstellung von Baumwolle (CO), Polyester (PET), Polypropylen (PP), Lyocell (CLY) und Viskose (CV). Das linke Bild zeigt den Energiebedarf, wobei erwartungsgemäß die synthetischen Fasern den höchsten Verbrauch aufweisen. Deutlich besser schneiden dabei die zellulosischen Chemiefasern ab, die hinsichtlich des Energiebedarfs sogar deutlich unter Baumwolle liegen. Die gleiche Reihung (mittleres Bild) ergibt sich, wenn die Emissionen von Treibhausgasen verglichen werden. Polyester und Polypropylen weisen die höchsten Emissionen auf, gefolgt von Baumwolle. Deutlich geringere Emissionen verursacht die Produktion von zellulosischen Fasern. Die relative große Differenz zwischen Viskose und Lyocell beruht auf unterschiedlichen Primärenergiequellen der jeweiligen Standorte. Im rechten Bild wird der extrem hohe Wasserbedarf zur Baumwollproduktion veranschaulich, der um mehr als eine Zehnerpotenz über den anderen Fasern liegt. Je nach Region und Effizienz der Bewässerungsanlagen kann der Wasserverbrauch bis auf $27000 \mathrm{~m}^{3} / \mathrm{t}$ ansteigen (Paulitsch et al. 2004).

Diese Daten zeigen, dass die Produktion von Fasern, auch Naturfasern, relativ hohe Auswirkungen auf die Umwelt hat. Eine Wiedergewinnung und Wiederverwendung von Fasern aus ökologischen Gesichtspunkten ist daher nahe liegend. Dies gilt auch dann, wenn Recyklatfasern zu- meist nicht wieder im selben Einsatzgebiet verwendet werden können, sondern auf einem qualitativ niedrigeren Niveau einer Nachnutzung zugeführt werden („Downcycling"). Diese Verlängerung des Lebenszyklus, bevor die Fasern schlussendlich entsorgt werden, kann im Hinblick auf die bei der Produktion verbrauchten Ressourcen einen nennenswerten Beitrag zur Schonung der Umwelt beitragen.

\section{Potentielle Abfallfraktionen zur Wiedergewinnung von Fasern}

\subsection{Beurteilung von faserhältigen Abfällen}

Wie oben erwähnt, finden sich Fasern in einer Vielzahl von Abfallfraktionen. Für ein Recycling von Fasern ist es in einem ersten Schritt notwendig, Abfälle zu finden, die sich als "Rohstoff“ zur Fasergewinnung eignen. Im Folgenden werden Parameter aufgelistet, die für die Beurteilung von potentiell interessanten Abfallströmen relevant sind:

- Existiert eine getrennte Sammlung für faserhältige Abfälle oder fallen bei Abfallaufbereitungsprozessen faserhältige Fraktionen an?

- Wie hoch ist die Faserkonzentration? Nahe liegender Weise sind möglichst hohe Werte anzustreben.

- Wie konstant ist die Faserkonzentration? Stark schwankende Anteile an Fasern erschweren die Aufbereitung.

- Wie ist die Faserzusammensetzung? In der Praxis kann nur ein einziges Fasermaterial vorliegen (z. B.: Produktions- abfälle) oder aber ein Fasermix aus zehn oder mehr Materialien (z.B.: gemischte Alttextilien).

- Wie viele nicht fasrige Komponenten sind vorhanden? Dies kann von einer einzigen Komponente (z. B.: Gummi in Fasern aus Altreifen) bis zu einer Vielzahl von organischen, metallischen und oxydischen Bestandteilen reichen (z. B.: Shredderleichtfraktion).

- Enthält die Abfallfraktion Stoffe, die die Aufbereitung erschweren? Harte oder metallische Komponenten müssen entfernt werden, bevor schnelllaufende Aggregate zur Aufbereitung eingesetzt werden können.

- Sind Stoffe enthalten, die eine potentielle Gefahr für die Umwelt darstellen? Gegebenenfalls müssen bei der Aufbereitung entsprechende Vorkehrungen getroffen werden bzw. müssen bestimmte Anwendungsgebiete ausgeschlossen werden.

\subsection{Altreifen}

Altreifen werden getrennt gesammelt und vielfach einer Verwertung zugeführt, zumal, gemäß der Richtlinie 99/31/EG, Altreifen nicht deponiert werden dürfen. Häufig werden zerkleinerte Altreifen als Ersatzbrennstoff in Zementwerken eingesetzt, wobei dies als Verwertungsverfahren gemäß 91/156/EWG einzustufen ist. Alternativ können Altreifen jedoch in einem mehrstufigen Prozess mittels Granulatoren und Schneidmühlen stufenweise zerkleinert werden. Dabei werden die Fraktionen Gummimehl, Stahl und Flusen 
erhalten. Gummimehle, deren Anteil ca. $65 \%$ beträgt, stellen hochwertige Produkte dar und können in vielen Bereichen (z. B. Sportplatzbau, Straßenbau, Dämmplatten, etc.) gewinnbringend vermarktet werden. Ein weiteres gewinnbringendes Produkt stellt die Stahlfraktion dar, deren Anteil bei rund $30 \%$ liegt, und direkt in die Stahlproduktion rückgeführt werden kann. Die rund 5 bis $10 \%$ Flusen, die die in PKW Reifen enthaltenen Cordfasern sowie nicht abgetrenntes, sehr feines Gummimehl enthalten, wurden bislang aufgrund des Eigenschaftsprofils, vor allem dessen sehr schlechtes Dosier- und Förderverhalten, primär thermisch verwertet. In einem Forschungsprojekt, das von 2004 bis 2006 von der Fa. GVG, heute ART (Asamer Rubber Technology) betrieben wurde, konnte gezeigt werden, dass die Fasern aus der Flusenfraktion zu einem marktfähigen Produkt für die Bitumenindustrie aufbereitet werden kann (Bartl et al. 2005). Die Zusammensetzung der Fasern liegt bei $50 \%$ Viskose, $30 \%$ Polyester und $20 \%$ Polyamid (Weisser und Czapay 1991). Die primär in PKW-Reifen verwendeten Fasern kommen ausschließlich in hochwertiger Cordqualität zum Einsatz, die, wie die Daten in Tabelle 1 zeigen, hinsichtlich ihres Eigenschaftsprofils und Preisniveaus deutlich über Fasern textiler Qualität liegen. Eine Wiederverwendung der Fasern aus Altreifen ist daher nahe liegend.

Die Projekterkenntnisse ermöglichen es ART nunmehr die Flusenfraktion aufzubereiten und gewinnbringend in der Bitumenindustrie abzusetzen (Bahardoust et al. 2006). Anstatt wie bislang Entsorgungskosten zu entrichten, kann nun ein Verkauferlös mit einem Recyclingprodukt erzielt werden.

\subsection{Altkleider}

Einer der wenigen Bereiche, in denen Altfasern relativ rein und homogen vorliegen, sind Altkleider. In Deutschland werden jährlich etwa 1 Million $t$ Bekleidung in Umlauf gesetzt. Mittels getrennter Sammelsysteme werden etwa 580.000 t erfasst, der Rest landet im Hausmüll (bsve 2001). Neben Altbekleidungen werden durch diese Sammlung auch Heimtextilien, wie Decken oder Leintücher, erfasst. Nach der Sammlung der Altkleider werden diese durch geschulte Fachkräfte von Hand sortiert. Dabei werden rund $50 \%$ tragfähige Bekleidung erhalten, die zum einen Teil in Second-Hand-Shops verkauft wird, zum anderen Teil als Spenden für Bedürftige zum Einsatz kommt. Diese Wiederverwer- tung der Bekleidung in ursprünglicher Form ist aus ökologischer, ökonomischer und sozialer Sicht vorteilhaft. Etwa 17\% der nicht mehr tragfähigen Bekleidung werden zu Putzlappen verarbeitet und rund $21 \%$ können zur Gewinnung von Reißspinnstoffen oder zur Papier- und Pappenherstellung verwendet werden. Schlussendlich müssen $12 \%$ deponiert oder thermisch verwertet werden (bsve 2001).

Die Sammlung und Sortierung von Altbekleidung trägt sich durch den Verkauf der aussortierten Altkleider selbst und wird nicht durch Abgaben oder Gebühren gestützt, wie dies bei anderen $\mathrm{Ab}$ fallgruppen üblich ist. Ein Gewinn ist jedoch nur mit rund $30 \%$ der gesammelten Ware zu erzielen. Damit müssen auch die Aufbereitung der übrigen Fraktionen und die Entsorgung der Reststoffe finanziert werden. Aufgrund der steigenden Kosten für die Entsorgung der Reststoffe (Verbrennung), wird die ökonomische Situation der Altkleidersammler immer schwieriger. Daher startete im August 2008 ein Forschungsprojekt unter der Leitung der TU Wien. Die Finanzierung erfolgt zum einen durch das Bundesministerium für Verkehr, Innovation und Technologie (BMVIT) im Rahmen der Programmlinie „Fabrik der Zukunft“ (Projekt Nummer 814961) sowie durch die Projektpartner HUMANA, $\mathrm{R}+\mathrm{M}$ und UEG. Ziel des Projektes ist es, ein mechanisches Aufbereitungsverfahren zu entwickeln, um denjenigen Teil der Altbekleidung wirtschaftlich aufzubereiten, der aktuell nur defizitär betrieben werden kann (Bartl und Marini 2008; Bartl und Haner 2009). Dabei sollen für Rezyklatfasern aus Altbekleidungen ähnliche, gewinnbringende Anwendungen gefunden werden, wie dies bei Fasern aus Altreifen bereits möglich ist.

\subsection{Shredderleichtraktion}

Zur Behandlung von sperrigen Abfällen wie Altautos, Herde, Waschmaschinen, Geschirrspüler aber auch leichter Mischschrott in Form von Fahrrädern und Bettgestellen haben sich Hammerbrecher schwerster Bauart, so genannte Shredder (Motek 1975), seit langem etabliert. Nach der Zerkleinerung werden die magnetisierbaren Teile (Eisen-Fraktion) sowie nicht-magnetisierbare Metalle (NE-Fraktion: Aluminium, Kupfer) zur Wiederverwertung abgetrennt. Neben den Metallfraktionen fällt jedoch ein Shredderabfall, die so genannte Shredderleichtfraktion (SLF), im Ausmaß von rund $25 \%$ (Geiger et al. 1993) an.
In Europa wird SLF großteils noch immer deponiert, jedoch gewinnen andere Verwertungsarten zunehmend an Bedeutung. Neben einem Deponierungsverbot für unbehandelte Anfälle übt die Altautorichtlinie 53/2000/EG auf die SLF großen Einfluss aus. In dieser Richtlinie werden seit 2006 verbindliche Quoten zum Altautorecycling festgesetzt, die 2015 noch weiter verschärft werden.

Aufgrund dieser hohen erforderlichen Recyclingrate ist eine Verwertung der SLF unumgänglich. Dabei können thermische Verfahren, die auf Verbrennung, Pyrolyse und Vergasung basieren, zum Einsatz kommen. Dabei wurden diese klassischen Verfahren von einigen Firmen für die Behandlung von SLF optimiert, wie etwa von EBARA („Twinrec“ Verfahren; Hirayama et al. 1995; Fujimura et al. 1997; Selinger et al. 2003), CTC Umwelttechnik („RESHMENT“ Verfahren; Schaub 2002; Aldo et al. 2004a und 2004b), Thermoselect (Thermoselect Verfahren; Kiss 1992; Drost et al. 2004; Yamada et al. 2004), Sustec Industries AG (,Schwarze Pumpe“ Prozess; Rabe et al. 1997; Anonymous 2001; Hauptmann et al. 2004; Buttker et al. 2005), Citron Holding AG („Oxyreducer“ Verfahren; Brueggler 2008) und ScanArc Plasma Technologies (PyroArc Verfahren). Die thermischen Verfahren verursachen relativ hohe Behandlungskosten. Parallel dazu haben sich mechanische Verfahren etabliert, die die SLF durch Zerkleinern, Sieben oder Sichten in weitere Fraktionen auftrennen. Durch die Abtrennung von Wertstoffen, primär Eisen- und NE-Eisen Metalle, kann ein zusätzlicher Gewinn erwirtschaftet werden. Je nach Verfahren fallen hauptsächlich Kunststoffgranulat, Inertmaterial und Flusen an. Mehrere Firmen haben diese bekannten mechanischen Verfahrensschritte für SLF optimiert. Anlagen werden von den Firmen Galloo („SRTL“ Verfahren; Vandeputte 1999; De Feraudy 2000 und 2007), VW/Sicon (Goldmann et al. 2007a, 2007b, 2007c und 2007d), TBS, UEG, Salyp, LSD („WESA“ Verfahren), Scholz und Cometsambre betrieben. Das bei der Aufbereitung erhaltene Kunststoffgranulat, das je nach Verfahren in Fraktionen unterschiedlicher Dichte getrennt sein kann, kann als Sekundärrohstoff verwendet werden, wie etwa als Reduktionsmittel bei der Stahlerzeugung im Hochofenprozess (Buergler und Perchtold 2004; Buergler 2008). Die anorganischen, vorwiegend mineralischen Bestandteile fallen in einer so genannten Inert- oder Sandfraktion an, die einer Deponierung zugeführt werden 
kann, da der TOC Gehalt unter $5 \%$ liegt. Je nach Art der Aufbereitung fällt eine so genannte Flusenfraktion an, die primär aus Fasern, Folien und PU-Schäumen besteht. Die Flusenfraktion wird entweder zur Entwässerung von Klärschlamm eingesetzt oder thermisch verwertet. Zur Erhöhung der ökonomischen Effizienz des Prozesses sind aber auch höherwertigere Anwendungen, analog zu Fasern aus Altreifen, für die Flusenfraktion anzustreben, bei denen die spezifischen Vorteile der Fasern ausgenützt werden können (Bartl et al. 2008).

\subsection{Sonstige faserhältige Abfälle}

Teppiche bestehen zumeist aus einem zweilagigen Polypropylengewebe mit einer Zwischenlage aus Styrol-Butadien-Gummi und Calciumkarbonat. Die Polfasern (Polyamid) sind in das Polypropylengrundgewebe getuftet. In der Literatur werden Aufbereitungsmethoden für sortenreine Teppichabfälle beschrieben (Wang 1995).

Eine getrennte Sammlung von Teppichabfällen wäre etwa beim Abbruch von Bürogebäuden oder deren Renovierung denkbar, auch wenn mit einer Verunreinigung durch Bauschutt zu rechnen ist.

Im Rahmen der textilen Verarbeitungskette fallen häufig faserhältige Produktionsabfälle, wie etwa Flusen aus Absauganlagen bei textilen Prozessen, an, die zumeist bereits relativ kurze, vereinzelte Fasern enthalten. Auch entstehen beispielsweise bei der Konfektionierung von Kleidungsstücken Stanz- oder $\mathrm{Zu}$ schnittreste, aus denen durch eine entsprechende Aufbereitung Fasern wieder gewonnen werden können. Diese Reststoffe liegen zumeist rein oder mit genau definierten Begleitmaterialien vor, wodurch ein Recycling vereinfacht wird. Da jedoch die Verarbeitung von Fasern und die Herstellung von Textilien zum überwiegenden Teil außerhalb der EU (z.B.: China) durchgeführt werden, unterliegt der entsprechende Umgang mit diesen Abfällen nicht dem EU Recht.

Eventuell könnte ein Recycling von Flusen Sinn machen, die bei Großwäschereien anfallen. So werden etwa in Deutschland jährlich 1 Million t Bekleidung in Umlauf gesetzt, wovon während des Gebrauchs durch Verschleiß, Waschen und Trocknen etwa $160.000 \mathrm{t}$ verloren gehen (Klatt 2001).

Es ist nicht auszuschließen, dass noch weitere interessante faserhältige Abfälle gefunden werden können. So

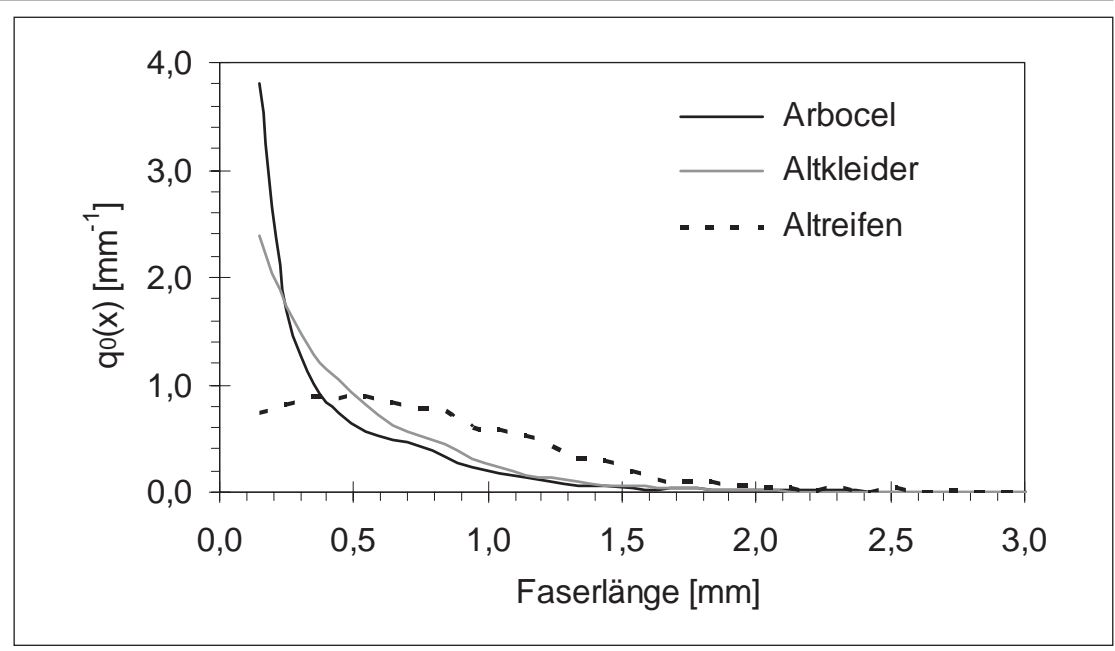

Abb. 2: Faserlänge von gemahlener Zellulose (Arbocel ${ }^{\circledR}$ ) und Fasern, die aus Altkleidern bzw. Altreifen rezykliert wurden (Bartl et al. 2005; Bartl und Haner 2009).

können durch geänderte gesetzliche Rahmenbedingungen und durch Etablierung neuer Recyclingtechnologien eventuell neue faserhältige Fraktionen entstehen. Nicht zuletzt beeinflussen Rohstoff- und Energiepreise massiv die ökonomischen Rahmenbedingen von Recyclingprozessen.

\section{Charakterisierung von Fasern}

Fasern aus Recyclingprozessen sind zumeist Kurzfasern und hinsichtlich ihrer Länge mit Flock zu vergleichen. Als Flock werden Kurzfasern bezeichnet, die nicht zum Verspinnen hergestellt werden (BISFA 2000). Üblicherweise liegen die Faserlängen im Bereich von 0,3 bis $5 \mathrm{~mm}$. Dieser Längenbereich ist weder mit klassischen Methoden der Partikelgrößenanalyse, noch mit textilen Charakterisierungsmethoden zu bestimmen. Bei jedem Aufbereitungsschritt von Fasern ist es jedoch notwendig, über eine entsprechende Charakterisierung, vor allem der Länge, zu verfügen, die eine qualitative Beurteilung des Produkts und des Aufbereitungsprozesses ermöglichen. Es zeigte sich, dass zur Bestimmung von Kurzfasern ein optisches Analysesystem (MorFi), das zur Zellstoffcharakterisierung entwickelt wurde (Passas et al. 2001; Tourtollet 2001), geeignet ist. Das Messgerät kann rasch und mit hoher Genauigkeit die Länge und den Durchmesser sowie die jeweilige Verteilungsdichte von Fasern zu bestimmen, die im Bereich von Flock bzw. Recyclingprodukten liegen (Bartl und Pico 2009; Bartl et al. 2005; Bartl et al. 2004).

Abbildung 2 zeigt die Faserlängenverteilung von Rezyklatfasern aus Altbekleidungen bzw. Altreifen nach einer Mah- lung in einer Feinschneidmühle (Bartl et al 2005; Bartl und Haner 2009), die mit dem MorFi System ermittelt wurden. Im Vergleich dazu wird die Längenverteilung von gemahlener Zellulose $\left(\right.$ Arbocel $\left.^{\circledR}\right)$ dargestellt, die als gängiges Additiv in der Baustoffindustrie eingesetzt wird. Dabei weist das Recyclingprodukt aus Altkleidern eine nahezu gleichartige Verteilungsdichtefunktion auf. Aufgrund einer anderen Faserzusammensetzung der Rezyklatfasern aus Altreifen (enthält keine Naturfasern) ist der Anteil an sehr kurzen Fasern $(<0,3 \mathrm{~mm})$ deutlich geringer.

Eine Charakterisierung der Fasern stellt eine Vorbedingung für einen erfolgreichen Recyclingprozess und für die Vermarktung des Recyclingproduktes dar. Neben der internen Prozesskontrolle kann das Recyclingprodukt hinsichtlich der Fasergeometrie genau charakterisiert werden und mit bereits am Mark befindlichen Faserprodukten verglichen werden. Dies stellt ein sehr wirkungsvolles Marketinginstrument dar

\section{Anwendungen für Rezyklatfasern}

\subsection{Voraussetzungen}

Die Wirtschaftlichkeit eines Recyclingprozesses für Fasern ist nur dann gegeben, wenn das hergestellte Produkt auch gewinnbringend vermarktet werden kann. Dies kann gelingen, wenn Anwendungen gefunden werden, in denen durch Verwendung des Recyclingproduktes entweder technische Vorteile erzielt werden oder eine Substitution derzeit gängiger Produkte möglich und wirtschaftlich ist. 


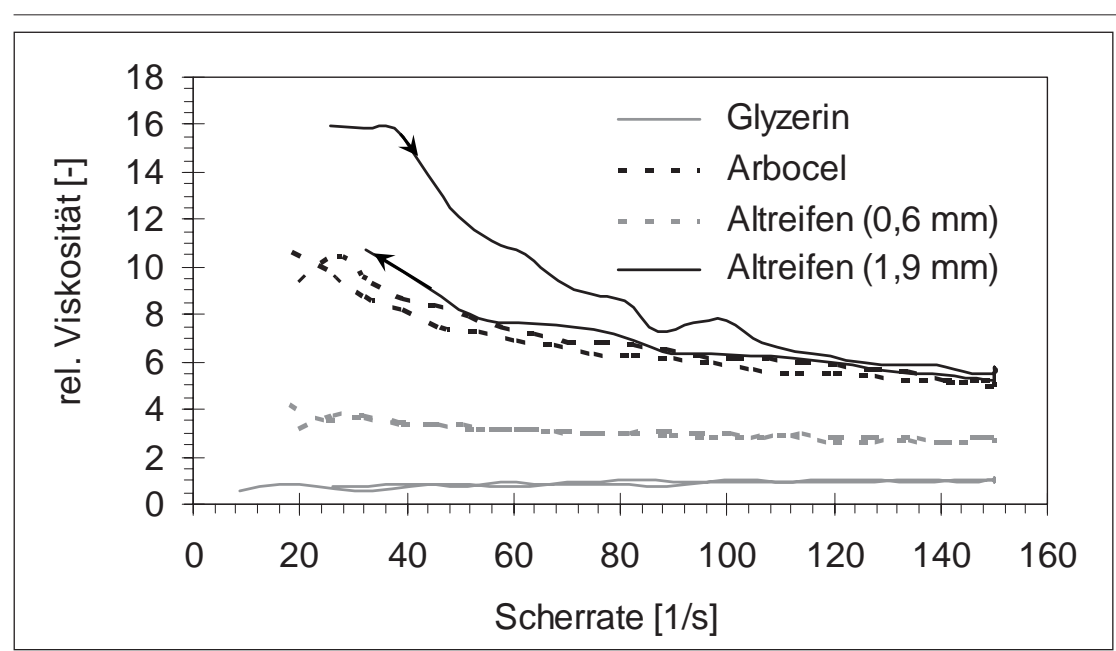

Abb. 3: Relative Viskosität bezogen auf Glyzerin von gemahlenem Zellstoff (Arbocel ${ }^{\circledR}$ ) und Rezyklatfasern aus Altreifen (mittlere Faserlänge: 0,6 und 1,9 mm) in Abhängigkeit zur Scherrate; Konzentration der Fasern jeweils 1 Massen \% (Bartl et al. 2004).

\subsection{Rezyklatfasern als Bitumenadditiv}

Bitumen ist der bestimmende Baustoff im Asphaltstraßenbau und unentbehrlich bei der Herstellung von Abdichtungsmaterialien für den Hoch- und Tiefbau. Der jährliche Bitumenverbrauch liegt in Österreich bei etwa 600.000 t und in Deutschland bei rund 3,4 Mio t. Fasrige Additive spielen im Bitumenbereichen eine wichtige Rolle. Die Nachteile von Rezyklatfasern aus Altreifen stellen die schwarze Färbung und der Gummigeruch dar. Diese Eigenschaften sind für Anwendungen im Bitumenbereich jedoch nicht störend. In Zusammenarbeit mit dem Institut für Straßenbau und Straßenerhaltung der TU Wien wurde daher eine gebrauchsverhaltensorientierte Bindemittelprüfung durchgeführt, um die Auswirkungen der Zugabe der gemahlenen Reifenflusen auf die Gebrauchseigenschaften und das Tieftemperatur, Ermüdungs und Verformungsverhalten sowie die Verarbeitbarkeit zu bestimmen (Bartl et al. 2005). Die Versuche mit modifiziertem Bitumen zeigten, dass deutliche Verbesserungen der Produktqualität von Asphaltschichten aus derartigen Produkten $\mathrm{zu}$ erwarten sind. Zwar verschlechtern sich die Tieftemperatureigenschaften um rund 2 bis $4^{\circ} \mathrm{C}$, jedoch werden gleichzeitig die Hochtemperatureigenschaften um rund $8{ }^{\circ} \mathrm{C}$ verbessert. In Summe kann somit die Gebrauchsspanne für das modifizierte Bindemittel um rund 4 bis $6^{\circ} \mathrm{C}$ vergrößert werden. Dies stellt eine deutliche Verbesserung des Bitumens dar, die sonst nur mit teuren Additiven erreicht werden kann. Üblicherweise kommen gemahlene Zellulose (z.B.: $\operatorname{Arbocel}^{\circledR}$ ), Thermoplaste (z. B.: ataktisches Polypropylen) oder Elas- tomere (z. B.: Styrolbutadienstyrol) zum Einsatz. Diese, teilweise sehr teuren Produkte, können, zumindest teilweise, durch Rezyklatfasern aus Altreifen oder auch anderen Quellen substituiert werden.

\subsection{Rezyklatfasern als Viskositätsmodifikator}

Eine Vielzahl von Produkten wird in flüssiger bzw. pastenförmiger Konsistenz appliziert, wie etwa Klebstoffe, Lacke oder Mörtel. Für viele dieser Produkte ist ein Newton'sches Fließverhalten, bei dem die Viskosität unabhängig von der Scherrate ist, unerwünscht. Pigmente und Füllstoffe, die in solchen Produkten enthalten sind, haben die Tendenz während der Lagerung zu sedimentieren. Gleichzeitig ist für eine problemlose Applikation, wie etwa Streichen oder Spritzen, eine niedrige Viskosität erforderlich. Durch Systeme, die einen durch Scherbeanspruchung verdünnend Effekt aufweisen, kann diesen Anforderungen Rechnung getragen werden. Im Falle, dass die Viskosität mit zunehmender Scherrate abnimmt, spricht man von Strukturviskosität bzw. pseudoplastischem Fließverhalten. Wenn zusätzlich die Abnahme der Viskosität auch noch zeitabhängig ist, spricht man von Thixotropie.

In der Praxis steht ein breites Spektrum an Additiven für diese Zwecke zur Verfügung, wobei unter anderem Polyurethane, Zellulosederivate (z. B.: Methylzellulose, Ethylhydroxypropylzellulose), feine Pulver (z. B.: pyrogene Kieselsäure, modifizierte Kaoline) und auch Fasern verwendet werden.

Es konnte bereits gezeigt werden, dass Rezyklatfasern das Newton'sche
Fließverhalten von Glyzerin in Richtung thixotrop ändern konnten (Bartl et al. 2006). In Abbildung 3 sind die Viskositätskurven von reinem Glyzerin und von Suspensionen $\left(1 \%\right.$ Arbocel $^{\circledR}$ bzw. Rezyklatfasern aus Altreifen; mittlere Länge 0,6 bzw. $1,9 \mathrm{~mm}$ ) verglichen.

Die prinzipielle Eignung von Rezyklatfasern als Viskositätsmodifikator ist gegeben, jedoch sind noch spezifische Anwendungsversuche notwendig, um eine gezielte industrielle Umsetzung zu ermöglichen.

\subsection{Weitere potentielle Anwendungsgebiete}

Der Bereich Betonbaustoffe umfasst ein sehr breites Gebiet. Unter Faserbeton versteht man im Allgemeinen alle Betone, die Fasern enthalten. Neben Faserart und Faseranteil unterscheidet man vor allem zwischen Betonen, die ein Gewebe bzw. ein Vlies enthalten und Betonen, die vereinzelte Kurzfasern enthalten. Durch Einsatz verschiedener Faserarten können bestimmte Betoncharakteristika erzielt werden. Faserzusatz kann etwa die Sprödigkeit verringern und die Formbeständigkeit erhöhen.

Zementfaserplatten bestehen aus einem relativ hohen Anteil an Fasern (rund 50\%) und Zement. Früher wurde dafür häufig Asbest eingesetzt, heute basieren die meisten Platten auf Zellulose-, Acryl-, Polypropylen- oder Polyvinylalkohol-Fasern. Ein Einsatz erfolgt vor allem im Bereich Wand und Deckenkonstruktionen. Derartige Platten zeichnen sich durch eine geringe Dichte und eine gute Schallund Wärmeisolierung aus.

Mörtel enthalten neben mineralischen Bindern, wie Kalk, Zement oder Gips, auch Polymere, Füllstoffe und Additive. Als Additive werden vielfach auch Fasern eingesetzt, wobei sowohl Lang- als auch Kurzfasern zum Einsatz kommen. Die Langfasern dienen primär zur Verstärkung des ausgehärteten Mörtels, während Kurzfasern, wie Arbocel $^{\circledR}$ oder Lignocel $^{\circledR}$, die Eigenschaften des feuchten Produkts (Viskosität) sowie dessen Wasseraufnahmevermögen beeinflussen.

\section{Schlussfolgerung und Ausblick}

Die Herstellung von Fasern verbraucht große Mengen an Energie und Rohstoffe, sodass eine Wiederverwendung von Fasern aus ökologischer Sicht anzustreben ist. Da Fasern sehr spezifische Eigenschaften aufweisen, im Speziellen eine hohe 
spezifische Oberfläche, kann ein Recycling auch ökonomisch sinnvoll sein. Es ist üblich, dass im Baustoffbereich vielfach faserhältige Additive eingesetzt werden. Eine Substitution dieser, teilweise teuren, Produkte durch Rezyklatfasern ist daher anzustreben.

Die Wiedergewinnung von Fasern ist aus verschiedenen Abfällen möglich, wobei zumeist Kurzfasern erhalten werden. Neben der Zusammensetzung des Fasermaterials sind vorhandene Verunreinigungen von großer Bedeutung. Problematische Stoffe, wie etwa Blei oder Chrom, müssen im Zuge der Aufbereitung abgetrennt werden, oder, wenn dies nicht möglich ist, sind Anwendungen zu wählen, bei denen die entsprechenden Grenzwerte eingehalten werden können.

Die Aufbereitung von Fasern verur- sacht Kosten, die jedoch durch Verzicht auf Wasser und den dadurch vermeidbaren hohen Trocknungskosten relativ gering gehalten werden können. Für die Zerkleinerungs- und Trennprozesse sind der Energiebedarf und der Verscheiß zu berücksichtigen, wobei mit Kosten in der Größenordnung von 100 bis $150 € / \mathrm{t} \mathrm{zu}$ rechnen ist. Der wirtschaftliche Vorteil besteht einerseits in der Vermeidung von hohen Entsorgungskosten, die, je nach Beschaffenheit des Abfalls, bis zu $200 € / t$ ausmachen können. Andererseits kann das Recyclingprodukt gängige Additive im Baustoffbereich substituieren, wodurch ein Erlös im Bereich von 200 bis $300 € / t$ möglich ist. In Summe besteht daher die Möglichkeit eines wirtschaftlichen Erfolges, wodurch andere Verwertungsschienen zurückgedrängt werden können.
Auch wenn einige Abfallströme als potentielle Rohstoffquellen zum Faserrecycling identifiziert werden konnten, so ist eine industrielle Umsetzung erst mit Fasern aus Altreifen gelungen. Für die weiteren Arbeiten sind Anwendungsversuche in verschiedenen Segmenten erforderlich, um dort marktfähige Produkte zu entwickeln. Dazu sind Forschungspartner aus der Industrie notwendig, die einerseits die Abfallwirtschaft repräsentieren und andererseits die potentiellen Anwender (z. B.: Baufirmen) verkörpern.

\section{Korrespondenz:}

TU Wien, Institut für Verfahrenstechnik

Getreidemarkt 9/166

1060 Wien

\section{LITERATUR}

Aldo J, Moergeli R, Dettwiler B (2004b) Processing of residual material and fumes, Patent EP0940166

Aldo J, Moergeli R (2004a) Method for removing metals from residual material, Patent EP1064982.

Anonymous (2001) Abfallvergaser, Gebrauchsmusterschrift DE20020240U1.

Bahardoust MH, Marini I, Neubacher F, Mihalyi B, Bartl A (2006) Verfahren zur Verwertung von Polymerarmierten Elastomerprodukten, Faserfraktion, bituminöses Mischgut, Baumaterial und Vorrichtung, Patent AT413355. Bartl A, Haner SA (2009) 12th International Conference on Process Integration, Modelling and Optimisation for Energy Saving and Pollution Reduction 10.-13. Mai 2009, Rom, Fiber Recovery from End-of-Life Apparel, zur Veröffentlichung angenommen.

Bartl A, Marini I (2008) Abfallforschungstage, 4.-5. Juni 2008, Hannover Altkleiderrecycling, Cuvillier Verlag, Göttingen, 14-24.

Bartl A, Pico D (2009) 9th International Conference on Chemical \& Process Engineering 10. -13. Mai 2009, Rome, Characterization of Short Fibers, zur Veröffentlichung angenommen

Bartl A, Hackl A, Mihalyi B, Wistuba M, Marini I (2005) Recycling of fibre materials for us in construction, Process Saf. Environ. Prot., 83 , B4, 351-358.

Bartl A, Mihalyi B, Marini I (2004) Application of renewable fibrous materials, Chemical and Biochemical Engineering Quarterly, 18, 1, 21 28.

Bartl A, Wistuba M, Spiegl M, Marini I, Bla R (2008) Verfahren zur Herstellung eines Zuschlagstoffs für die Herstellung von Baumaterialien, Patent AT504885.

Bartl A, Mihalyi B, Madtha L, Marini I (2006) Chemical Industry and Environment $V$, Ferdinand Berger \& Söhne GmbH, „Recycled Fibers as Viscosity Modifiers“; 1104 - 1111. BISFA (2000), Terminology of man-made $\mathrm{fi}$ bers.

Brueggler M (2008) Method for gasification of waste in a rotary hearth furnace and furnace for gasification of waste, Patent EP2003395. bsve (2001) Bundesverband Sekundärrohstoffe und Entsorgung, Textilrecycling, Zahlen - Daten - Fakten.

Buergler T, Perchtold G (2004) DEPOTECH, Leoben; 14.-26. November 2004; in: „Abfalund Deponietechnik, Altlasten, Abfallwirtschaft", Die Bedeutung der Post-ShredderTechnologie für die ökologische und ökonomische Verwertung von Altfahrzeugen,

\section{$505-510$}

Buergler T (2008) Depo Tech, Leoben, 12.-14. November 2008; in: „Abfall- und Deponietechnik, Altlasten, Abfallwirtschaft . K. Lorber, G. Kreindl, P. Müller, D. Sager, K. Wruss (Hrg..); VGE Verlag, Kunststoffe im Hochofenprozess Vorbereitung, Umsetzung und Ergebnisse, 297 302.

Buttker B, Giering R, Schlotter U, Himmelreich B, Wittstock K (2005) Full scale industial recovery trials of shredder residue in a high temperature slagging-bed-gasifier in Germany, Bericht SVZ.

CIRFS (2008) The International Rayon and Synthetic Fibres Committee, Information on Man-Made Fibres - 44th volume, Bruxelles. De Feraudy H (2000) Method and installation for separating all categories of polymer materials, Patent EP1058609.

De Feraudy H (2007) Method for preconcentration of organic synthetic materials derived from shredding of end-of-life durable goods, Patent US7252251.

Drost U, Eisenlohr F, Kaiser B, Kaiser W, Stahlberg R (2004) Report on the operating trial with automotive shredder residue (ASR), Geneva.

Fujimura H, Hirayama Y, Fujinami S, Nagato S, Hirose T, Oshita T, Irie M, Takano K, Fukuda T (1997) Method and apparatus for treating wastes by gasification, Patent EP0803562. Geiger T, Baumgartner H, Seifert H, Dorn IH, Knopf H (1993) Possibilities of thermal treatment of light fractions from shredded automo bile scrap, Chemie Ingenieur Technik, 65, 4, 444-446.

Goldmann D, Dunnen B, Knust M (2007a) Anlage und Verfahren zur Aufbereitung von Shredder Rückständen, Patent EP1337341. Goldmann D, Dunnen B, Knust M (2007b) Anlage und Verfahren zur Aufbereitung von Shredder Rückständen und Verwendung einer e zeugten Granulatfraktion, Patent EP1332001. Goldmann D, Dunnen B, Knust M (2007c) Anlage und Verfahren zur Aufbereitung von Shredder Rückständen und Verwendung einer erzeugten Flusenfraktion, Patent EP1333931. Goldmann D, Dunnen B, Knust M (2007d) Anlage und Verfahren zur Aufbereitung von Shredder Rückständen und Verwendung einer erHauptmann W, Seifert W, Pocher B, Kamka F (2004) Gasifying waste, e.g. clarified sludge and refuse, comprises using fixed bed gasifier unit, entrained bed pressure gasifier unit, GuD power station, and synthesis gas evaluator, $\mathrm{Pa}$ tent DE10031501.
Hirayama Y, Oshita T, Tame C, Nagato S, Hirose T, Miyoshi N, Toyoda S, Hosoda S, Fujinami S, Takano K (1995) Method of and app ratus for fluidized-bed gasification and melt combustion, Patent EP0676464.

Kiss G (1992) Process for rendering usable disposal products, Patent DE4130416.

Klatt S (2001) Entsorgungsverhalten des Bürgers ist entscheidend für das Textilrecycling, Bericht Bundesverband Sekundärrohstoffe und Entsorgung.

Koslowski H (2000) Dictionary of Man-Made Fibers, International Business Publishers $\mathrm{GmbH}$, Frankfurt/Main.

Motek H (1975) Hartzerkleinerungsmaschine für neue Verfahrenstechniken, Aufbereitungs Technik (1960-1989), 8, 378-392.

Norm (1999) DIN 60001-1, Textile Faserstoffe; Teil 1: Naturfasern und ihre Kurzzeichen, Deutschland.

Passas R, Voillot C, Tarrajat G, Caucal G,

Khelifi B, Tourtollet GEP (2001) Morfi as a novel technology for morphological analysis of fibers, Recents Progres en Genie des Procedes, 15, 78, 259-264.

Patel M, Shen L (2008) Comparative Environmental Assessment of Man-made Cellulose

Fibres, 47. Chemiefasertagung, Dornbirn.

Paulitsch K, Baedecker C, Burdick B

(2004) Am Beispiel Baumwolle: Flächennut-

zungskonkurrenz durch exportorientierte Land-

wirtschaft, Wuppertal papers.

Rabe W, Hauptmann W, Buttker B, Duerlich

M, Koschmieder K (1997) Production of material made of waste, Patent DE19621921.

Schaub M (2002) ISWA Frühjahrstagung, 11.

April 2002, Dübendorf, Rohstoffliches Recyc-

ling am Beispiel der RESH-Verwertung mit dem RESHMENT Prozess.

Selinger A, Steiner C, Shin K (2003) International Automobile Recylcing Congress, 12.-14. März 2003, Genf, TwinRec - Bridging the Gap of Car Recycling in Europe.

Tourtollet GEP (2001) More reliable, more ac curate, and less expensive, Ipw 1, 10-14. Vandeputte A (1999) Method and apparatus for separating polymeric materials, Patent EP0918606.

Wang Y (1995) Reuse of carpet industria waste for concrete reinforcement, RILEM Proc. 27, 297-306.

Weisser H, Czapay M (1991) Twisting systems for tyre cord production, Chemiefasern/Textilindustrie, 41, 1224-1234.

Yamada S, Shimizu M, Miyoshi F (2004) Thermoselect Waste Gasification and Reforming Process, JFE Giho 3, 20-24. 